\title{
Determination of Sexual Problems of Turkish Patients Receiving Gynecologic Cancer Treatment: a Cross-sectional Study
}

\author{
Basak Demirtas $^{1 *}$, Gul Pinar ${ }^{1,2}$
}

\begin{abstract}
Background: The present study aimed to determine the prevalence and types of sexual problems of Turkish patients receiving gynecologic cancer treatment. Materials and Methods: A cross-sectional convenience sample of 168 women completed the Index of Female Sexual Function (IFSF) and a Patient Identification Form in a

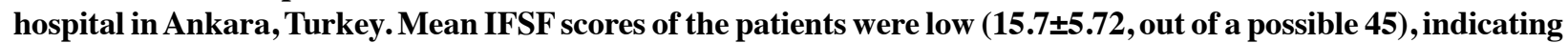

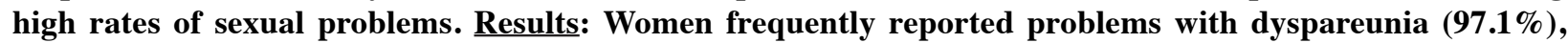
vaginal dryness $(\mathbf{9 7 . 6 \%})$, decreased sexual desire $(\mathbf{9 1 . 1 \%})$, and difficulties of sexual arousal $(92.9 \%)$ related with the cancer treatment process. They reported increased sexual problems following the period of treatment as compared to before treatment $(\mathbf{p}<\mathbf{0 . 0 5})$. Sexual dysfunction was associated with low educational and income levels, advanced age, TAH-BSO-LND surgery (total abdominal hysterectomy-bilateral salphingoopherectomylymph node dissection), experiencing side effects of chemotherapy, receiving chemotherapy in addition to surgery and radiotherapy $(C T+R T+$ Surgery $)$, and having a large number of chemotherapy cycles $(p<0.05)$. Conclusions: Patients hoped for and expected counseling from healthcare professionals about their sexual functioning in relation to cancer treatments. Nurses and physicians can help to improve the overall quality of life for gynecologic cancer patients through sexual counseling.
\end{abstract}

Keywords: Gynecologic cancer treatment - physicians- nurses - sexuality - sexual counseling

Asian Pac J Cancer Prev, 15 (16), 6657-6663

\section{Introduction}

One out of almost six cancer cases seen in women throughout the world is a gynecological cancer. According to the data of the International Agency for Research on Cancer-IARC, gynecological cancers are responsible for $19 \%$ of the new cancer cases (Sankaranarayanan and Ferlay, 2006). In Turkey, ovarian cancers are fifth most common while the endometrial cancers are fourth most common among women (National Cancer Institute, 2007; Globocan, 2008).

Treatment of gynecologic malignancies generally requires surgical intervention, radiotherapy, chemotherapy, and/or hormonal therapy, all of which have a potential to affect sexuality (Lee et al., 2006; Stilos et al., 2008; Eker and Açıkgöz, 2011). Literature shows that 50\% of women with gynecologic cancers report sexual dysfunction (Audette and Waterman, 2010). Type, stage, recency of diagnosis, disease recurrence, conditions specific to the treatment, and culture and beliefs are included in the factors determining the effect of the cancer and its treatment on sexuality (Krebs and Marrs, 2006; Nieman et al., 2006; Hordern and Annette, 2007).

Gynecological cancer TAH-BSO-LND operation, side effects of chemotherapy, treatment mode including chemotherapy in addition to surgery and radiotherapy $(\mathrm{CT}+\mathrm{RT}+$ Surgery) have also been shown to be associated with poor sexual functioning (Kaplan et al., 1999; Yilmaz and Eryılmaz, 2004; Demirezen, 2006; Incrocci and Jensen, 2013). It has also been demonstrated that sexual dysfunction is common and chronic in patients who undergo radical hysterectomy (Shell, 2007). This is consistent with other studies that have demonstrated that surgical interventions can cause sexual dysfunction (Kaplan et al., 1999; Yılmaz and Eryılmaz, 2004).

Research examining the sexual problems of patients who receive gynecologic cancer treatment is also sparse in Turkey (Corney et al., 1993; Aslan et al., 2008). Therefore little is known about sexual problems of patients who receive gynecologic cancer treatment in Turkey (Corney et al., 1993; Yılmaz and Eryılmaz, 2004; Erenel and Kitiş, 2011; Akkuzu and Ayhan, 2013). Our study attempts to address this gap in the literature.

\section{Materials and Methods}

\section{Aim}

The study aimed to determine the prevalence and types of sexual problems of patients who receive gynecologic cancer treatment. 


\section{Methods}

This descriptive and cross-sectional study was conducted with patients at the Outpatient Chemotherapy Unit in a hospital in Ankara, Turkey. All participants were undergoing chemotherapy treatment for their cancer at the time of the study. The population consists of the patients that applied to the Ankara Women's Health Training and Research Hospital, Gynecologic Oncology Outpatient Chemotherapy Unit within a one year period to receive chemotherapy $(n=300)$. The percentage of patients who were approached who declined to participate is $4.5 \%$ $(\mathrm{n}=8)$.

Based on a power analysis, 168 participants were need to achieve a power (power: $1-\beta$ ) of $80 \%$. Data collection process was continued till the required number was achieved (August-December, 2010). A cross-sectional convenience sample of 168 women completed the Index of Female Sexual Function (IFSF) and a Patient Identification Form in a hospital in Ankara, Turkey. The inclusion criteria of study were as follows: being 18 years old or older, having a partner, 4 months had passed from the diagnosis and surgical treatment as a minimum, having received at least 2 cycles of the chemotherapy, not being in the terminal period, having had sexual intercourse within last four weeks, and being willing to participate in the study. Four different types of cancer (ovarian, endometrial, cervix, vulva) were represented among the participants of this study.

More than half of the patients $(56 \%)$ are in the age group 41-55, and one-fourths (25\%) is in the age group 56-68 (mean age: $49.23 \pm 8.91$, min: 26 , max:68). The period of marriage for more than half $(53.6 \%)$ varies between 19 and 34 years. Majority have stated that they have middle-level income (72.6\%). Majority are graduates of primary school (81\%). Although not shown in the table, three-quarters live in a core family (75\%), $82.1 \%$ have children between 2 and 4 in number. All of the patients had insurance.

\section{Instruments}

Data was collected through a two-part survey, which included a) a Patient Identification Form and b) IFSF. The Patient Identification Form consisted of 43 items related to the socio-demographic characteristics of patients, characteristics of the diseases and its treatment, sexual life before and after the treatment, and request for information on the sexual life.

The IFSF, which has been developed by Kaplan et al. (1999) to consist of 9 questions, each item investigates the sexual functioning of the woman within the last 4 weeks. The reliability and validity testing of the scale in Turkish was performed by Yllmaz and Eryılmaz, in 2004. The study performed by Yilmaz and Eryilmaz (2004) has indicated the Cronbach's Alpha reliability coefficient to be 0.82 . The average cut-off point of the scale is $30(\min =5, \max =45)$. Low scores are associated with low sexual functioning. The scale is formed up of three sub-dimensions, namely, discomfort during sexual intercourse, frequency of sexual intercourse/libido and sexual satisfaction.

\section{Ethical considerations}

The study was conducted in compliance with principles of Helsinki Declaration. Approval was obtained from the Ethical Board of the Women's Health Training and Research Hospital in order to conduct the study. Women were informed about the study by the researchers prior to study and the ones who agreed to participate were enrolled in the study.

\section{Data analyses}

Data were analyzed using SPSS 15.0 (SPSS Inc, Chicago, IL). Percentage distributions were used to analyze the socio-demographic and descriptive characteristics of the patients; means and standard deviations were used to calculate the scale scores. $P$ values $<0.05$ were accepted as statistically significant. In order to analyze the relation between the dependent variables and independent variables, Student t test, Chi-square test, and One-way ANOVA tests were used. For following-up tests with more than 2 groups, the Bonferroni multiplecomparison correction was used. In determining the risk factors for sexual problems, a multinominal logistic regression analysis was used.

\section{Results}

\section{Participant characteristics}

As seen in Table 1, ovarian cancer was the most common diagnosis $(77.4 \%)$. For more than half of patients $(62.5 \%)$, the period during which the patients had symptoms but did not have a cancer diagnosis was less than one year. The surgical procedure performed on $97 \%$ of the patients was TAH-BSO-LND. The treatment mode in $67.9 \%$ was surgery together with chemotherapy. The number of cycles of chemotherapy was 3 to 6 in $79.2 \%$. Chemotherapy protocol was taxol+carboplatin in $58.3 \%$ of the patients, and $44.6 \%$ of the patients were in Stage 3 while $25 \%$ were in Stage 4 . Side effects related to treatment were seen in $97 \%$ of the patients, with the most frequent side effects including fatigue and weakness $(92 \%)$, hair loss $(91 \%)$, nausea and vomiting $(85 \%)$, pain $(82 \%)$. Thirty-three point three percent had another chronic disease in addition to cancer, such as hypertension $(48.2 \%)$, diabetes $(35.7 \%)$, or arthritis (16.1\%) (Table 1).

As seen in the Table 2, while the rate of having sexual problems when healthy was $16.1 \%$, the rate during the treatment became $94.6 \%$. While the rate of the patients stating that they have a poor sexual life when healthy was $3.6 \%$, the rate after the treatment increased to $76.7 \%$. Nearly a fourth of the women (22.6\%) reported that they had sex 3 or more times per week when they were healthy; following treatment, no one reported having sex 3 or more times per week. Similarly, $66.1 \%$ of women reported having sex 1-3 times per week when healthy, and only $30.4 \%$ reported having sex 1-3 times per week following treatment (Table 2).

The rate of patients who did not receive information about their sexual life in relation to the cancer treatment was $81 \%$ (Table 1). Among those who did receive information, the source of information was the physician 
Table 1. Sexual Function Scores According to Some Sariables

\begin{tabular}{|c|c|c|c|c|}
\hline & $\mathrm{N}$ & $\%$ & $\begin{array}{c}\text { IFSF }(15.66 \pm 5.72) \\
\text { Mean } \pm \text { SD }\end{array}$ & Statistics F/t \\
\hline \multicolumn{5}{|c|}{ Socio-demographic characteristics } \\
\hline \multicolumn{5}{|l|}{ Age } \\
\hline $26-40$ & 32 & 19.0 & $18.41 \pm 7.98$ & \multirow{3}{*}{$\begin{array}{l}F=4.800^{\mathrm{a}} \\
\mathrm{p}<0.05\end{array}$} \\
\hline $41-55$ & 94 & 56.0 & $15.11 \pm 4.99$ & \\
\hline $56-68$ & 42 & 25.0 & $14.81 \pm 4.60$ & \\
\hline \multicolumn{5}{|l|}{ Educational level } \\
\hline Primary education & 136 & 81.0 & $14.03 \pm 5.52$ & \multirow{2}{*}{$\begin{array}{l}\mathrm{t}=5.547 \\
\mathrm{p}<0.05\end{array}$} \\
\hline$\geq$ High school & 32 & 19.0 & $16.14 \pm 5.85$ & \\
\hline \multicolumn{5}{|c|}{ Marital period (years) } \\
\hline 4 to 18 & 30 & 17.9 & $19.67 \pm 9.375$ & \multirow{3}{*}{$\begin{array}{c}\mathrm{F}=10.280^{\mathrm{a}} \\
\mathrm{p}<0.05\end{array}$} \\
\hline 19 to 34 & 90 & 53.6 & $15.07 \pm 3.951$ & \\
\hline 35 to 39 & 48 & 28.5 & $14.27 \pm 4.428$ & \\
\hline \multicolumn{5}{|l|}{ Economic status } \\
\hline Low & 36 & 21.4 & $14.46 \pm 3.66$ & \multirow{3}{*}{$\begin{array}{c}\mathrm{F}=11.338^{\mathrm{a}} \\
\mathrm{p}<0.05\end{array}$} \\
\hline Medium & 122 & 72.6 & $18.53 \pm 7.70$ & \\
\hline High & 10 & 6.0 & $20.40 \pm 11.07$ & \\
\hline \multicolumn{5}{|l|}{ Number of children } \\
\hline 1 & 22 & 13.1 & $18.00 \pm 8.58$ & \multirow{3}{*}{$\begin{array}{l}F=2.757^{a} \\
p>0.05\end{array}$} \\
\hline 2 to 4 & 138 & 82.1 & $15.46 \pm 5.20$ & \\
\hline 5 to 8 & 8 & 4.8 & $12.40 \pm 0.894$ & \\
\hline \multicolumn{5}{|l|}{ Family type } \\
\hline Core family & 127 & 75.5 & $18.87 \pm 7.31$ & \multirow{2}{*}{$\begin{array}{l}t=0.465 \\
p>0.05\end{array}$} \\
\hline Broad family & 41 & 24.5 & $16.25 \pm 7.22$ & \\
\hline \multicolumn{5}{|l|}{ Employment } \\
\hline Yes & 7 & 4.2 & $17.14 \pm 5.17$ & $\mathrm{t}=0.489$ \\
\hline No & 161 & 95.8 & $15.60 \pm 5.74$ & $\mathrm{p}>0.05$ \\
\hline Medical Characteristics & & & & \\
\hline Cancer type & & & & \\
\hline Endometrial & 30 & 17.9 & $15.30 \pm 4.88$ & $\mathrm{~F}=0.408^{\mathrm{a}}$ \\
\hline Ovarian & 130 & 77.4 & $15.84 \pm 4.00$ & $\mathrm{p}>0.05$ \\
\hline Cervical & 8 & 4.7 & $14.13 \pm 3.79$ & \\
\hline Period of diagnosis & & & & \\
\hline$\leq 12$ months & 105 & 62.5 & $17.35 \pm 6.92$ & $t=7.724$ \\
\hline$>12$ months & 63 & 37.5 & $15.53 \pm 5.12$ & $\mathrm{p}<.05$ \\
\hline Operation & & & & \\
\hline TAH & 5 & 3.0 & $16.00 \pm 6.33$ & $\mathrm{t}=8.840$ \\
\hline TAH-BSO-LND & 163 & 97.0 & $15.20 \pm 3.25$ & $\mathrm{p}<0.05$ \\
\hline Treatment mode & & & & \\
\hline $\mathrm{CT}^{\mathrm{b}}$ & 4 & 2.4 & $17.66 \pm 6.69$ & $\mathrm{~F}=13.541^{\mathrm{a}}$ \\
\hline $\mathrm{CT}+$ Surgery & 114 & 67.9 & $15.00 \pm 3.41$ & $\mathrm{p}<0.05$ \\
\hline $\mathrm{CT}+\mathrm{RT}^{\circ}+$ Surgery & 50 & 29.8 & $12.75 \pm 3.50$ & \\
\hline Side effects chemother & erapy & & & \\
\hline Yes & 163 & 97.0 & $15.53 \pm 5.41$ & $t=9.726$ \\
\hline No & 5 & 3.0 & $19.80 \pm 12.53$ & $\mathrm{p}<0.05$ \\
\hline Cycles of Chemothera & apy & & & \\
\hline 3 to 6 & 133 & 79.2 & $18.09 \pm 8.63$ & $\mathrm{~F}=6.328^{\mathrm{a}}$ \\
\hline 7 to 10 & 24 & 14.3 & $15.65 \pm 5.77$ & $\mathrm{p}<0.05$ \\
\hline$>10$ & 11 & 6.5 & $14.63 \pm 3.20$ & \\
\hline Chronic diseases & & & & \\
\hline Yes & 56 & 33.3 & $15.30 \pm 5.23$ & $t=6.312$ \\
\hline No & 112 & 66.7 & $17.38 \pm 6.58$ & $\mathrm{p}<0.05$ \\
\hline Information Related to & Sexua & Life & & \\
\hline I was informed & 32 & 19.0 & $15.21 \pm 4.12$ & $\mathrm{t}=3.214$ \\
\hline I was not informed & 136 & 81.0 & $15.16 \pm 4.05$ & $\mathrm{p}>0.05$ \\
\hline Sexual life information & on sour & ee $(n=3$ & & \\
\hline Physician & 27 & 84.3 & $14.24 \pm 5.53$ & $\mathrm{t}=8.317$ \\
\hline Nurse & 5 & 15.6 & $16.33 \pm 6.72$ & $\mathrm{p}<0.05$ \\
\hline Seeking more informa & ation $\mathrm{r}$ & lated $t$ & sexual life & \\
\hline Yes & 125 & 74.4 & $15.43 \pm 5.81$ & $t=9.326$ \\
\hline No & 43 & 25.6 & $17.25 \pm 6.94$ & $\mathrm{p}<0.05$ \\
\hline The favorite source of & $f$ infor & hation & elated to sexual life & $(n=125)^{d}$ \\
\hline Physician & 114 & 91.2 & $14.53 \pm 5.32$ & $t=10.312$ \\
\hline Nurse & 11 & 8.8 & $17.36 \pm 6.88$ & $\mathrm{p}<0.05$ \\
\hline
\end{tabular}

in $84.3 \%$ of cases. Seventy-four point four percent of the patients wished to be informed about the impact of treatment on their sexual life, and of those, $91.2 \%$ wished to get this information from the physician (Table 1). The subjects related to sexual life that patients wish to be informed about included whether or not sexual intercourse would be harmful during the treatment process $(60.8 \%)$, whether or not they would have pain during sexual intercourse $(35.2 \%)$, whether or not infections would develop (18.4\%), whether or not the wound site would be damaged (17.6\%), whether or not pain and lack of desire during intercourse is normal $(11.2 \%)$, and whether or not they had caught the cancer from their partner (7.2\%). Although not shown in the table, some patients believed that it is possible that their disease advanced because of sexual intercourse and that it was possible that the disease was transmitted from their spouses.

The relationship between socio-demographic characteristics and IFSF scores of patients with gynecological cancer

In this study, the IFSF mean score was $15.66 \pm 5.72$ (min: 10, max: 42). Scores on the IFSF were associated with age, educational level, income level, and length of marriage $(p<0.05)$. Scores on the IFSF were not associated with number of children, family types and employment ( $>>0.05)$ (Table 1).

Results of follow-up tests (Bonferroni) showed that there was a statistically significant difference between the mean IFSF score in the 26 to 40 age group and in the 56 to 68 age group $(\mathrm{F}=11.622, \mathrm{p}<0.01)$, with older age being associated with worse sexual functioning. According to follow-up tests results it was also identified that there was a statistically significant difference between the mean IFSF score among those with shorter marriages (4-18 years) as compared to longer marriages (35-39 years) ( $\mathrm{F}=10.496$, $\mathrm{p}<0.05)$, with shorter marriages being associated with less dysfunction. Follow-up tests results also demonstrated that patients with low income level had poorer sexual functioning than those with medium or high income levels $(\mathrm{F}=12.979, \mathrm{~F}=16.33 \pm 4.07, \mathrm{p}<0.01)$.

The relationship between medical characteristics and IFSF scores of patients with gynecological cancer

Sexual functioning was associated with experiencing chemotherapy side effects, having another chronic condition in addition to cancer and also associated with type of operation, number of cycles of chemotherapy, and treatment mode $(\mathrm{p}<0.05)$. Sexual function scores were not associated with cancer type $(\mathrm{p}>0.05)$ (Table 1$)$.

Follow-up tests (Bonferroni) results demonstrated that patients receiving chemotherapy in addition to surgery and radiotherapy had higher prevalence of sexual dysfunction than those receiving other treatment modes $(\mathrm{F}=10.367, \mathrm{~F}=14.21 \pm 3.86, \mathrm{p}<0.05)$. Results of follow-up tests also showed that patients who received between 3 and 6 chemotherapy cycles had lower prevalence of sexual dysfunction than those who received between 7 and 10 or than 10 chemotherapy cycles $(\mathrm{F}=11.475, \mathrm{~F}=15.30 \pm 3.92$, $\mathrm{p}<0.05)$.

Compared to their functioning prior to treatment, the patients in the study had poorer sexual lives following the period of treatment, the frequency of their sexual intercourses diminished, and they had increased sexual problems. These differences were statistically significant $(\mathrm{p}<0.05)$ (Table 2). 
Table 2. Comparison of the Sexual Life Before and After the Treatment

\begin{tabular}{|c|c|c|c|c|c|}
\hline \multirow[t]{2}{*}{ Sexual life } & \multicolumn{2}{|c|}{$\begin{array}{c}\text { Before } \\
\text { the treatment }\end{array}$} & \multicolumn{2}{|c|}{$\begin{array}{c}\text { After } \\
\text { the treatment }\end{array}$} & \multirow[t]{2}{*}{$\begin{array}{c}\text { Statistic } \\
\qquad \mathrm{x}^{2} / \mathrm{p}\end{array}$} \\
\hline & $\mathrm{N}$ & $\%$ & $\mathrm{~N}$ & $\%$ & \\
\hline \multicolumn{6}{|l|}{ Sexual problem } \\
\hline Yes & 27 & 16.1 & 159 & $94.6^{\mathrm{b}}$ & 7.665 \\
\hline No & 141 & 83.9 & 9 & 5.4 & $\mathrm{p}<0.05^{\mathrm{a}}$ \\
\hline \multicolumn{6}{|l|}{ Sexual life } \\
\hline Poor & 6 & 3.6 & 129 & 76.7 & 6.840 \\
\hline Medium & 41 & 24.3 & 21 & 12.5 & $\mathrm{p}<0.05^{\mathrm{a}}$ \\
\hline Good & 121 & 72.1 & 18 & 10.8 & \\
\hline \multicolumn{6}{|c|}{ Frequency of sexual intercourse } \\
\hline$>3$ per week & 38 & 22.6 & 0 & 0 & 7.033 \\
\hline 1-3 times per week & 111 & 66.1 & 51 & 30.4 & $\mathrm{p}<0.05^{\mathrm{a}}$ \\
\hline Rarely & 19 & 11.3 & 117 & 69.6 & \\
\hline
\end{tabular}

${ }^{\mathrm{a} M c N e m a r}$ 's Chi-square $\left(\mathrm{x}^{2}\right)$ test was used; ${ }^{\mathrm{b}}$ Vaginal dryness, dyspareunia, difficulties of sexual arousal, decreased sexual desire

As seen in Table 2,94.6\% of the patients reported that they had sexual problems after the cancer treatment. Of those who experiences sexual problems, the problems reported were decreased sexual desire $(91.1 \%)$, vaginal dryness (97.6\%), difficulties of sexual arousal (92.9\%), and dyspareunia $(97.1 \%)$. Of those who reported sexual problems $59.7 \%$ indicated that their problems occurred following the surgery and 34\% indicated that they occurred following the chemotherapy. However, $32.1 \%$ of those having sexual problems reported that they did not share these problems with their spouses.

No significant relationship was found between having adequate information about their sexual life and their mean sexual functioning scores $(\mathrm{p}>0.05)$; however, women who sought more sexual information related to their sexual life reported more sexual problems than women who did not seek out such information, and they wished to get these information from physicians as opposed to nurses $(\mathrm{p}<0.05)$ (Table 1).

Accordingly; sexual problems of the patients between the ages of 56 to 68 is $57 \%$ greater than those who are younger $(\mathrm{CI} ; 0.96-1.98, \mathrm{p}=0.002)$. The frequency of sexual problem of the patients who are married for longer periods of time $(\mathrm{CI} ; 1.85-5.22, \mathrm{p}=0.009)$ is 2.54 times greater than those who are married for shorter periods of time (Table 3 ).

As the patient's level of education increases, the frequency of sexual problem decreases $63 \%$ and it is significant as well $(\mathrm{CI} ; 0.57-4.53, \mathrm{p}=0.003)$. The frequency of sexual problem of the patients with high economic status is 3.22 times less than those with both low and medium economic status $(\mathrm{CI} ; 3.11-9.40, \mathrm{p}=0.001)$. Furthermore, when performing regression analysis between the frequencies of chronic disesases and sexual problem of the patients, statistically significant relation was found (CI; $0.89-2.37, \mathrm{p}=0.003$ ). It was found that sexual problems were seen more intensely with the increasing period after the diagnosis. The frequency of sexual problem of the patients with the increasing period after the diagnosis is 2.28 times greater than patients with the shorter period after the diagnosis (CI; 1.61-4.53, $\mathrm{p}=0.005$ ). Additionally, the frequency of sexual problem of the patients with increasing number of chemo cycles is 2.01 times more than patients with decreased number of chemo cycles (CI; 0.86-1.60, $\mathrm{p}=0.004)$. Finaly, the
Table 3. Regression analysis of variables affecting sexual problems

\begin{tabular}{|c|c|c|c|c|}
\hline Variables & OR & $t$ & $p$ & $95 \%$ CI IFSF \\
\hline \multicolumn{5}{|l|}{ Age } \\
\hline $26-40$ & 1.00 & 2.35 & 0.002 & - \\
\hline $41-55$ & 1.28 & & & $0.77-1.76$ \\
\hline $56-68$ & 1.57 & & & $0.96-1.98$ \\
\hline \multicolumn{5}{|l|}{ Education } \\
\hline Primary education & 1.00 & 2.43 & 0.003 & - \\
\hline$\geq$ High school & 1.63 & & & $0.57-4.53$ \\
\hline \multicolumn{5}{|l|}{ Economic status } \\
\hline Low & 1.13 & 2.56 & 0.001 & - \\
\hline Medium & 2.17 & & & $1.48-2.62$ \\
\hline High & 3.22 & & & $3.11-9.40$ \\
\hline \multicolumn{5}{|l|}{ Marital period (years) } \\
\hline 4 to 18 & 1.87 & -3.31 & 0.009 & - \\
\hline 19 to 34 & 1.98 & & & $0.77-1.76$ \\
\hline 35 to 39 & 2.54 & & & $1.85-5.22$ \\
\hline \multicolumn{5}{|l|}{ Period of diagnosis } \\
\hline$\leq 12$ months & 1.00 & -2.67 & 0.005 & - \\
\hline$>12$ months & 2.28 & & & $1.61-4.53$ \\
\hline \multicolumn{5}{|c|}{ Side effects chemotherapy } \\
\hline Yes & 1.00 & -3.86 & 0.000 & - \\
\hline No & 3.34 & & & $2.25-7.64$ \\
\hline \multicolumn{5}{|c|}{ Cycles of chemotherapy } \\
\hline 3 to 6 & 0.80 & 3.12 & 0.004 & $0.48-1.42$ \\
\hline 7 to 10 & 1.00 & & & $0.67-1.26$ \\
\hline$>10$ & 2.01 & & & $0.86-1.60$ \\
\hline \multicolumn{5}{|l|}{ Chronic diseases } \\
\hline Yes & 1.00 & -3.15 & 0.003 & - \\
\hline No & 1.27 & & & $0.89-2.37$ \\
\hline
\end{tabular}
logistic regression was used

frequency of sexual problem of the patients who did not experience the side effects of the chemotherapy is 3.34 times less than those who experience the side effects of the chemo $(\mathrm{CI} ; 2.25-7.64, \mathrm{p}=0.000)$ (Table 3$)$.

\section{Discussion}

Cancer survivorship is increasing due to more effective cancer treatment; thus, people are living longer with the effects of cancer treatment. As a result there is an increase of issues related to the sexuality, as an important element affecting the quality of life, among cancer survivors (Keskin and Gümüş, 2011; Bober and Varela, 2012).

In our study, mean IFSF scores of the patients are rather below $(15.66 \pm 5.72)$ compared to the total score possible (min: 10, max: 42). It was determined that patients with longer periods of marriage, those in the older age group, and those with lower educational and income levels had a significantly higher prevalence of sexual dysfunction than those with shorter periods of marriage, those in younger age groups, and those with higher educational and income levels. Additionally, longer period since the diagnosis and presence of another chronic disorders are significantly predictive of poor sexual functioning. This is consistent with other studies conducted in Turkey, which also found a significantly higher prevalence of sexual dysfunction in the presence of older age, lower educational level, unemployment status (Aslan et al., 2008; Erenel and Kitiş, 2011; Akkuzu et al., 2014). In the literature, consistent with the findings of the current study, it is reported that a large proportion of patients with gynecologic cancers suffer sexual problems (Barton-Buckle and Gustason, 
2007; Reis et al., 2010).

The findings in this study showed that sexual functioning is poorer when patients have experienced a larger number of cycles of chemotherapy and when they report side effects of the chemotherapy. It was also found that the most common side effects encountered by patients (in order of frequency reported) included fatigue and weakness, hair loss, nausea-vomiting, and pain. It has been reported in the literature that fatigue, decreased sexual desire, dyspareunia, difficulty achieving orgasm, depression, anxiety, marital or partner difficulties, and altered perception of body image are common side effects of chemotherapy (Cull et al., 1993; Wenzel et al., 2003; Akkuzu, 2012).

In the study, it was found that the differences between sexual function scores according to the cancer types were not statistically significant. However, the rates of non-ovarian cancer were very low in this sample, so the nonsignificant results may have been due to a lack of power. Shell (2007) showed that patients with ovarian cancer had more sexual problems than those with other types of gynecologic cancer, perhaps because the ovarian cancer manifests itself in the advanced stage and has a more insidious course.

In this study, it was found that patients who underwent TAH-BSO-LND had more sexual problems than patients who underwent solely TAH-BSO. Similarly, Corney et al. (1993) reported that sexual dysfunction was common and chronic in patients who underwent radical hysterectomy. This is consistent with other studies that have demonstrated that surgical cancer interventions can cause sexual dysfunction in between $20 \%$ and $100 \%$ percent of patients (Kaplan et al., 1999; Y1lmaz and Eryılmaz, 2004). According to the information obtained in our study, among patients who experienced sexual problems, commonly reported problems included dyspareunia (97.1\%), vaginal dryness $(97.6 \%)$, decreased sexual desire $(91.1 \%)$ and difficulties with sexual arousal $(92.9 \%)$ related to the treatment.

Among those who reported sexual problems in our study, more than half of the patients stated that the problems started following the surgery, and more than a quarter stated that started following the chemotherapy. Another important consideration is establishing the most appropriate time to address sexual issues. Health care professionals should begin discussing psychosexual issues related to diagnosis and treatment during consent for treatment process (Taylor and Davis, 2006; Stilos et al., 2008). The patient's focus at the time of diagnosis usually is on treatment and survival. The discussion of sexual side effects may be overlooked or forgotten if it is presented during this time. Informed consent should include a discussion about the effects of treatment on sexuality (Katz, 2005; Krebs and Marrs, 2006).

In our study, almost one-fifth of the patients reported that they had sexual problems even when they were healthy. In Turkey, as in most cultures, many healthy women without any physical diagnosis or disease have sexual problems (Yılmaz and Eryılmaz, 2004; Reis et al., 2010; Erenel and Kitiş, 2011). Because Turkey is a traditional societ, sexual experience before marriage is unacceptable particularly for young women, and women often engage in sex so as to meet the requirements of men (Aslan et al., 2008; Erenel and Kitiş, 2011). There are studies showing that patients with satisfactory sexual lives before treatment tend to have satisfactory sexual lives after treatment and are able to successfully cope with the effects of the cancer treatment on their sexual life (Corney et al., 1993; Helstrom, 1994; Yılmaz and Eryılmaz, 2004).

It was noted in our study that more than a quarter of the women having sexual problems did not share their sexual problems with their spouses. Under the influence of the social, cultural, and religious beliefs in Turkey, sexuality continues to a subject is considered a taboo (Aslan et al., 2008; Reis et al., 2010). Gynecologic and obstetric issues are seen as women's issues, not to be discussed with men. It is often viewed as embarrassing for partners to have knowledge about women's health issues (Yılmaz and Eryılmaz, 2004; Erenel and Kitiş, 2011; Olsson et al., 2012). In their review, Wenzel et al. (2003) emphasized that men need more information about what is happening with their partner's body during cancer treatments, and they need to be prepared for anticipated changes in their sexual life. According to another study conducted in the USA, for individuals who are cancer survivors, an unsupportive relationship tend to increase distress (Barton-Buckle and Gustason, 2007). However, a supportive relationship tend to decrease psychological distress for patients with cancer (Ananth et al., 2003). Therefore, ensuring the participation of the spouses in the treatment process of the woman starting from the time of cancer diagnosis is important in regards to the woman's ability to cope with problems during the healing process (Burns et al., 2007; Shell, 2007; Sekse et al., 2010).

The majority of the patients in our study stated that they did not get information about their sexual lives and that they needed information in this area. The patients wished for information about whether or not sexual intercourse would be harmful during treatment, whether or not they would feel pain during intercourse, whether or not they would have infections, whether or not the wound site would be damaged, and whether or not pain and lack of desire during intercourse is normal. Ananth et al. (2003) concluded that, if provided early after diagnosis, explanation about potential sexual problems that may be encountered during the course of the disease may give patients confidence to discuss such issues as they occur, thus avoiding embarrassment or aggravation of problems later in their illness.

According to the findings of our study, women who sought more sexual information reported more sexual problems. Women particularly wished to get sexual information from physicians. Patients with greater sexual problems may prefer physicians because of their need for sexual help extends beyond counseling and into the realm of medical intervention. Physicians and nurses who work in oncology settings may function in several roles, providing expert clinical, educational, emotional, and supportive care to cancer patients (Hordern and Annette, 2007; Chao et al., 2014). Kim et al. (2011) determined that Sexual Health Care Scale-Attitude scores which measure attitudes regarding sexual health care were high for Korean 
nurse group with participation in sexual health training.

In the present study, some patients believed that it is possible that their disease advanced because of sexual intercourse and that it was possible that the disease was transmitted from their spouses. Similarly, Cull et al. (1993) found out that more than $25 \%$ of the women with gynecological cancer blamed their partners for transmitting the disease. Womens' perceived causes of diseases may increase their risk for anxiety. Communication between the patient and clinician about sexual issues offers the health care professional the ability to understand a patient's concerns and offer appropriate treatments (Audette and Waterman, 2010; Carter et al., 2013).

In conclusion, the study examined sexual problems of patients who receive gynecologic cancer treatment. The results of this study indicate that majority of the patients diagnosed with gynecologic cancers face sexual problems related to treatment and expect counseling from healthcare professionals in this area. Increased education for gynecologic oncology nurses and physicians about the importance of and strategies for communication about sexuality could result in better care for gynecologic cancer survivors.

The study findings may contribute to the understanding of nurses' and physicians' role in sexual information delivery and, subsequently, in patient education and counseling in oncology practice in the countries with no sexual health consultation services for gynecologic cancer patients. Additional research is needed to determine which methods can be used to provide consultation and information as well as the effectiveness of these methods.

\section{References}

Akkuzu G, Talas MS, Ortac F (2014). Functional status in Turkish women with gynecological cancer. Asian Pac J Cancer Prev, 15, 2045-9.

Akkuzu G, Ayhan A (2013). Sexual functions of Turkish women with gynecologic cancer during the chemotherapy process. Asian Pac J Cancer Prev, 14, 3561-4.

Akkuzu G (2012). An evaluation of the quality of life of women undergoing chemotherapy due to a diagnosis of a gynecological oncological disease. Asian Pac J Cancer Prev, 13, 1277-80.

Ananth H, Jones L, King M, Tookman A (2003). The impact of cancer on sexual function: a controlled study. Palliative Med, 17, 202-5.

Aslan E, Beji NK, Güngör I, Kadioglu A, Dikencik BK (2008). Prevalence and risk factors for low sexual function in women: a study of 1009 women in an outpatient clinic of a university hospital in Istanbul. J Sex Med, 5, 2044-52.

Audette C, Waterman J (2010). The sexual health of women after gynecologic malignancy. J Mıdwıfery Wom Heal, 55, 357-62.

Barton-Buckle M, Gustason CJ (2007). Sexuality in women with cancer. Nurs Clin N Am, 42, 685-96.

Bober SL, Varela VS (2012). Sexuality in adult cancer survivors: challenges and intervention. J Clin Oncol, 20, 3712-9.

Burns M, Costello J, Ryan-Woolley B, Davidson S (2007). Assessing the impact of late treatment effects in cervical cancer: an exploratory study of women's sexuality. Eur $J$ Canc Care, 16, 364-72.

Carter J, Stabile C, Gunn A, Sonoda Y (2013). The physical consequences of gynecologic cancer surgery and their impact on sexual, emotional, and quality of life issues. $J$ Sex Med, 10, 21-34.

Cayan S, Akbay E, Bozlu M, et al (2004). The prevalence of female sexual dysfunction and potential risk factors that may impair sexual function in Turkish women. Urol Int, 72, 52-7.

Chao YH, Wang SY, Hsu TH, Wang KWK (2014). The desire to survive: the adaptation process of adult cancer patients undergoing radiotherapy. Jpn J Nursing Sci, [Epub ahead of print].

Corney RH, Crowther ME, Everett H, Howells A, Shepherd JH (1993). Psychosexual dysfunction in women with gynaecological cancer following radical pelvic surgery. $\mathrm{Br}$ J Obstet Gynaecol, 100, 73-8.

Cull A, Cowie VJ, Farquharson DIM, et al (1993). Early stage cervical cancer: psychosocial and sexual outcomes of treatment. Br J Cancer, 68, 1216-20.

Demirezen E (2006). Evaluation of woman sexuality in primary health care. STED , 15, 80-4.

Eker F, Açıkgöz F (2011). The impact of cancer and its treatment on sexual desire, satisfaction and functioning: findings from an exploratory study in rural Turkey. Eur J Cancer Care 20, 769-75.

Erenel AŞ, Kitiş Y (2011). A study on determination of women's sexual function. Türkiye Klinikleri J Gynecol Obst, 21, 251-9.

GLOBOCAN (2008). Cancer Incidence and Mortality Worldwide: International Agency for Research on Cancer. Available from: http://globocan. iarc.fr. Accessed $10 \mathrm{Feb}$ 2014.

Helstrom L (1994). Sexuality after hysterectomy: a model based on quantitative and qualitative analysis of 104 women before and after subtotal hysterectomy. J Psychosom Obstet Gynecol, 15, 219-29.

Hordern AJ, Annette FS (2007). Communicating about patient sexuality and intimacy after cancer: mismatched expectations and unmet needs. Med J Australıa, 186, 224-7.

Incrocci L, Jensen PT (2013). Pelvic radiotherapy and sexual function in men and women. J Sex Med, 10, 53-64.

Kaplan SA, Reis RB, Kohn IJ, et al (1999). Safety and efficacy of sildenafil in postmenopausal women with sexual disfunction. J Urology, 53, 481-6.

Katz A (2005). The sounds of silence: sexuality information for cancer patients. J Clin Oncol, 23, 238-41.

Keskin G, Gümüş BA (2011). Turkish hysterectomy and mastectomy patients - depression, body 1mage, sexual problems and spouse relationships. Asian Pac J Cancer Prev, 12, 425-32.

Kim S, Kang HS, Kim JH (2011). A sexual health care attitude scale for nurses: development and psychometric evaluation. Int J Nurs Stud, 48, 1522-32.

Krebs L, Marrs JA (2006). What should i say? talking with patients about sexuality issues. Clin J Oncol Nurs, 10,313-5.

Lee SJ, Schover LR, Partridge AH, et al (2006). American Society of Clinical Oncology recommendations on fertility preservation in cancer patients. J Clin Oncol, 24, 2917-31.

National Cancer Institute (2007). Sexuality and reproductive issues health. Available from: http://www. Cancer. Gov/ cancer topics/pdq/supportivecare/sexuality/health Professional.

Nazik E, Arslan S, Nazik H, et al (2012) Anxiety and symptom assessment in Turkish gynecologic cancer patients receiving chemotherapy. Asian Pac J Cancer Prev, 13, 3129-33.

Nieman CL, Kazer R, Brannigan RE, et al (2006). Cancer survivors and infertility: a review of a new problem and novel answers. J Support Oncol, 4, 171-8.

Olsson C, Berglund AL, Larsson M, Athlin E (2012). Patient's sexuality- A neglected area of cancer nursing? Eur J Oncol 
Nurs, 16, 426-31.

Reis N, Beji KN, Coskun A (2010). Quality of life and sexual functioning in gynecological cancer patients: results from quantitative and qualitaty data. Eur J Oncol Nurs, 14, 137-46.

Sankaranarayanan R, Ferlay J (2006). Worldwide burden of gynaecological cancer: the size of the problem. Best Pract Res Clin Obstet Gynaecol, 20, 207-25.

Sekse RJT, Raaheim M, Blaaka G, Gjengedal E (2010). Life beyond cancer: women's experiences 5 years after treatment for gynaecological cancer. Scand J Caring Sci, 24, 799-807.

Shell JA (2007). Including sexuality in your nursing practic. Nurs Clin N Am, 42, 515-29.

Simon B, Lee SJ, Partridge AH, Runowicz CD (2005). Preserving fertility after cancer. CA: A Can J Clin, 55, 211-28.

Stilos K, Doyle C, Daines P (2008). Addressing the sexual health needs of patients with gynecologic cancers. Clin J Oncol Nurs, 12, 457-63.

Taylor B, Davis S (2006). Using the extended PLISSIT model to address sexual healthcare needs. Nurs Stand, 21, 35-40.

Wenzel L, VergoteI, Cella D (2003). Quality of life in patients receiving treatment for gynecologic malignancies: special considerations for patient care. Int J Gynecol Obstet, 83, 211-29.

Yılmaz C, Eryılmaz H (2004). Validity/reliability study of female sexual function index (IFSF). Androloji Bülteni, 18, 275-6. 